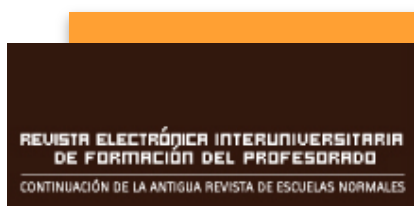

http://revistas.um.es/reifop

http://www.aufop.com/aufop/revistas/lista/digital

Bernabé, M.M. (2015). Organización intercultural en el aula de música de Secundaria. Revista Electrónica Interuniversitaria de Formación del Profesorado, 18 (3), 13-24.

DOI: http://dx.doi.org/10.6018/reifop.18.3.182971

\title{
Organización intercultural en el aula de Música de Secundaria
}

María del Mar Bernabé Villodre

Universidad de Valencia

\section{Resumen}

La diversidad cultural ha venido reclamando una igualdad efectiva desde finales del siglo $X X$, que se ha traducido en una revisión de contenidos curriculares de la Educación Secundaria Obligatoria. Desde la asignatura de Música se ha considerado posible un planteamiento que garantice la adquisición de la interculturalidad, gracias a que sus contenidos conceptuales más teóricos y los aspectos más prácticos de ella permiten el desarrollo de los diferentes elementos constitutivos de la educación intercultural. Este artículo muestra cómo una determinada organización de la educación musical puede contribuir al diálogo intercultural, como base del intercambio y, por tanto, de la interculturalidad.

Palabras clave

Interculturalidad; Música; Educación Secundaria; educación intercultural.

\section{Intercultural classroom organization teaching Music in Secondary Education}

\begin{abstract}
Cultural diversity has been calling for effective equality since the late twentieth century, which has resulted in a revised curriculum of compulsory Secondary Education. Thus, the Music subject has been considered a possible approach that ensures the acquisition of multiculturalism because of its conceptual contents as well as its practical aspects. These
\end{abstract}

\section{Contacto:}

María del Mar Bernabé Villodre, maria.mar.bernabe@uv.es, Universidad de Valencia, Facultad de Magisterio, Departamento de Didáctica de la Expresión Musical, Plástica y Corporal. Despacho 4.16. Avda. Taronger, 4. 46022 Valencia 
allow the development of different elements of intercultural education. This article shows how a particular organization of music education can contribute to intercultural dialogue as the basis of trade and, therefore, of multiculturalism.

\section{Key words}

Interculturalism; Music; Secondary Education; intercultural education.

\section{Introducción}

La presencia de diferentes culturas en el territorio nacional desembocó en sucesivos cambios curriculares desde las últimas décadas del siglo XX hasta esta segunda década del nuevo siglo, debido a las exigencias de una educación de calidad y en igualdad por parte del alumnado extranjero. Realmente, la construcción de un proyecto educativo que reconozca todas las culturas, las incluya y garantice su éxito escolar es muy complejo (Etxebarria, Karrera y Murua, 2010). Teóricamente, todas las asignaturas de Educación Secundaria Obligatoria (ESO) tenían que ofrecer contenidos de calidad para todos los educandos. En esta línea, la asignatura de Música fue la que más cambios presentó, y, debido a éstos y a las características intrínsecas de su propia práctica interpretativa y educativa, se convirtió en el centro neurálgico de la actividad intercultural del centro de ESO; ya que es una poderosa herramienta de transmisión de valores (De Moya, Hernández y Hernández, 2010) y puede considerarse el "producto" principal que plasma las creencias y sentimientos de una cultura. De manera que, desde este supuesto, puede convertirse en el centro neurálgico del trabajo intercultural (Ruiz, 2007; Cruz Roja Española, 2009).

El centro educativo tiene que convertirse en un espacio de participación real y efectiva de las culturas minoritarias, de expresión e intercambio cultural, para desarrollar una sociedad respetuosa con la pluriculturalidad. Esa diversidad cultural debe aprovecharse para conseguir un currículo interculturalizado (Leiva, 2011), que evite la mera inclusión de contenidos culturales "diferentes" con la única intención de garantizar que esa diversidad cultural sí se ha tenido en cuenta en los currículos. Aunque no puede olvidarse que la ESO coincide con la problemática adolescencia que se une al "problema" de la diversidad cultural (Pliego y Valero, 2011). De hecho, gran parte de los altercados acontecidos en el aula se deben más a cuestiones relacionadas con la sobrecarga hormonal que a los choques culturales: es el momento de construir la propia identidad y la falta de reconocimiento a la de la minoría cultural puede llevarle a rechazarla, lo que dificultará su proceso educativo y formativo.

En respuesta a la realidad sociocultural, la legislación planteó cambios centrados en una inclusión de contenidos conceptuales; sin embargo las actitudes y los valores de tipo intercultural no quedaban realmente reflejados junto a esos contenidos, en cuanto a procedimientos, criterios de evaluación, objetivos y actitudes. Entonces, se puede afirmar que la educación obligatoria ha reducido la educación intercultural al ámbito cognitivo (Touriñán, 2008), ya que sólo se han incluido contenidos culturales entendidos como la representación totalitaria de la cultura "del otro"; pero se han obviado los valores, el trabajo de la aceptación y el hecho de que el respeto a la diversidad no es más que el camino hacia la igualdad de derechos. De modo que para conseguir esos objetivos e intenciones interculturales es indispensable formar al profesorado, que debe ser consciente de que no basta con incluir contenidos culturales "del otro" sino que debe realizar las adaptaciones pertinentes en todos los sentidos. Leiva (2011) considera que esta formación debe ser teórica y práctica para poder establecer relaciones con el estudio de casos 
particulares que les permitirá comprender la aportación de los inmigrantes en el ámbito laboral, cultural y humano (Barquín, Alzola, Madinabeitia y Urizar, 2010).

En posteriores epígrafes se mostrará cómo superarse la interpretación musical de otros pueblos para completarse con un trabajo teórico comparado y una organización instrumental y del material, enfocados desde la educación intercultural en aras de conseguir la interculturalidad planteada en la legislación vigente.

\section{Revisión teórica}

\section{Aproximación al concepto educativo intercultural desde el contexto pluricultural español}

Se puede afirmar que la Historia Mundial es la historia de las migraciones (FETE-UGT, 2011). La Humanidad está acostumbrada a las migraciones porque la búsqueda de mejores condiciones de vida es inherente al ser humano (Ruiz y Merino, 2009); incluso podría considerarse que la perspectiva dinámica de la cultura y las culturas que introduce la interculturalidad (Fundación Secretariado Gitano, 2007) se perdió con el paso de los siglos. No obstante no debería obviarse que la diversidad es la norma (Colectivo Yedra, 2009), es decir, es más común que la unicidad, ya que todos somos diferentes incluso dentro de una pretendida homogeneidad. Tal como afirma Beriain (2013), los conflictos actuales parecen surgir más bien del choque dentro de las civilizaciones que por las que vienen; lo que debería contribuir a clarificar que todas las culturas son resultado de fusiones con otras que muestran su heterogeneidad.

En España se trató de evitar la homogeneización con una revalorización de los elementos tradicionales autóctonos a través del currículo educativo para lograr que el alumnado se convirtiese en un miembro de pleno derecho de la sociedad de la que formaba parte, aunque costó mucho incluir otros elementos que reflejasen la presencia de otras culturas presentes en el aula. De hecho, esa dirección comentada ha sido continuada con la LOE, desde 2006, que comprende la asignatura de Música como un bien cultural que refleja la historia y permite recuperar y afianzar la identidad cultural (Lorenzo y Espejo, 2011).

La llegada masiva de familias de inmigrantes supuso la demanda de una educación de calidad para todos, fuese cual fuese su cultura de pertenencia. Entonces se inició un proceso de reflexión y análisis que trajo consigo importantes consecuencias en la educación (Pérez y Sarrate, 2013), tales como la modificación curricular; sin embargo no todas las asignaturas modificaron sus contenidos ni registraron cambios en cuanto al trabajo de valores y actitudes relacionadas con la diversidad cultural. En este sentido, fueron asignaturas como Música o Ciencias Sociales las que presentaron cambios significativos. Aunque, por encima de todo cambio, un proceso educativo en estos contextos pluriculturales no tendrá garantías de éxito si no cuenta con un desarrollo de contenidos actitudinales, acordes con los conceptos y procedimientos trabajados. Desde la educación intercultural, se puede considerar que la diferenciación de contenidos conceptuales, procedimentales y actitudinales es de gran relevancia; aunque el uso de las nuevas tecnologías de la información y la comunicación aumentará los beneficios de cualquier intervención intercultural, como afirman Santos, Lorenzo y Priegue (2013).

El centro educativo quedaría convertido en un espacio de encuentro, de enriquecimiento, de intercambio, de exploración y de reflexión, porque al ser el principal centro de formación-educación podrá contribuir a que su diversidad cultural sea vista como un factor positivo (Pérez y Sarrate, 2013). Si queda comprendido como centro intercultural, deberá 
facilitar la integración del alumnado y de sus familias (FETE-UGT, 2009), y contribuirá a que ese espacio de resolución se extienda fuera del mismo. El centro educativo será considerado, así, un lugar en el que autóctonos e inmigrantes tomen conciencia de que deben mostrarse receptivos ante sus respectivas culturas; aunque por encima de cualquier otra consideración, debería contarse con que uno de los objetivos principales de la educación intercultural es dotar al alumnado de herramientas para construir su propia identidad.

Pero, ¿por qué es conveniente escoger un modelo educativo intercultural para la clase musical? Porque la educación intercultural favorece el diálogo, la convivencia, el sentimiento de igualdad, y el respeto a la diferencia (González, 2010); también prepara para el desarrollo de una convivencia pacífica, gracias al reconocimiento del otro y a la elección libre de valores que se realiza (Touriñán, 2008). Además, educar interculturalmente supone aceptación, predisposición hacia "el otro" (De Moya, Hernández y Hernández, 2010), y respuestas solidarias y tolerantes. Aunque básicamente, la importancia de seguir una línea educativa intercultural se debe a que sus principales características responden a los cuatro pilares educativos (UNESCO, 2006): enseñar a saber-conocer, enseñar a hacer, enseñar a vivir juntos, y enseñar a ser.

Touriñán (2008) considera que el reto de la interculturalidad consiste en formar al individuo como un ser capaz de combinar la cultura propia con aquella que tiene próxima y desplazarse de una a otra sin problemas. Aquí la Música justifica su importancia: los estilos musicales en la ESO permiten profundizar en la música desde una perspectiva comparada (elementos prestados y asimilados como propios, y así sucesivamente). Es decir, que la Música muestra las relaciones entre culturas y se muestra como fruto de la interacción cultural que pregona la educación intercultural, que implica interacción, intercambio, interdependencia, reconocimiento, respeto... Entre sus objetivos está la construcción de una cultura compartida que muestre los elementos de cada una de las culturas en contacto, de forma que son "nuestros" y no "míos" o "tuyos". Se trataría de crear un espacio social nuevo y conjunto regido por nuevas normas. No obstante sólo podrá construirse una cultura común si se reflexiona sobre la propia: de hecho la interculturalidad es concebida como una propuesta educativa reflexiva (Leiva, 2008). Y en esta dirección reflexiva, la Música es ejemplo e instrumento de enriquecimiento y reflexión porque invita al diálogo y esto es el elemento más importante para el proceso educativo intercultural (Sales, 2012): por ejemplo, en España, la música de Granados muestra toda la fuerza de la cultura andaluza, pero gran parte de esos elementos son resultado de fusiones y evoluciones fruto de contactos culturales derivados de invasiones o migraciones vividas en siglos pasados.

El enriquecimiento que aporta el proceso educativo intercultural sólo será posible cuando se apueste por el diálogo, la cooperación y el intercambio (Leiva, 2008). Es esta opción intercultural la que permitirá enfrentarse a los retos educativos de cualquier sociedad pluricultural; ya que ésta reconoce la diversidad cultural y prepara al alumnado para hacerle frente. De manera que se debe intentar educar interculturalmente en el aula musical de ESO porque tanto la etapa como la materia permiten desarrollar cómodamente los principios que la UNESCO (2006) considera característicos de la educación intercultural: posibilita una educación de calidad para todos, respeta la identidad cultural del educando, le provee de conocimientos culturales y actitudes necesarias para participar activamente en la sociedad de acogida, y le provee de los conocimientos y actitudes necesarias para contribuir al respeto y al entendimiento entre los diferentes grupos culturales de una nación.

La interculturalidad implica la "voluntad de" y un compromiso moral (Touriñán, 2008), puesto que si se educa tomando como punto de partida la ampliación de contenidos conceptuales, éstos no van a garantizar la comprensión del otro, ni las relaciones positivas y 
afectivas con él. Sin embargo si se toma como excusa la interpretación de una partitura africana para comentar los elementos que ésta tiene en común con la música propia (clásica, popular o tradicional), la Música se convertirá en una herramienta de reflexión, cambio e intercambio, y entonces sí se mejorará la relación con el otro y se labrará el camino para lograr la interculturalidad.

\section{La educación intercultural en las aulas de Música españolas}

En ESO, la asignatura de Música tiene unas características que la convierten en un espacio idóneo para trabajar interculturalmente, consideración compartida por autores como Samper (2011). Este punto de vista puede considerarse imprescindible hoy día si se parte del hecho de que la educación intercultural llevará al entendimiento cultural caracterizado por el conocimiento, las interrelaciones, el autoconocimiento y el replanteamiento de la cultura propia. Así pues, la Música puede considerarse el principal instrumento para trabajar la interculturalidad porque todas las culturas se expresan musicalmente (Gonzállez, 2010), e incluso goza de un papel privilegiado de transmisora de información y de acontecimientos relevantes de las diferentes comunidades. Además a todo esto se suma el hecho de que en esta aula todo el alumnado se encuentra presente, incluso aquellos que precisan refuerzo en otras materias, lo que la convierte en un punto de encuentro que debe aprovecharse para garantizar el diálogo cultural; puesto que la música nace gracias a las aportaciones de diferentes pueblos, como muestra la música flamenca (Escudero, 2012).

La clase de Música con su insistente trabajo sobre la música tradicional, véanse trabajos como los de Gómez (2011) que trabajan músicas tradicionales de todo el mundo, incita a la reflexión cultural y ayuda a ahondar en la cultura propia. $Y$ ese ahondar y reflexionar debe enfocarse hacia la similitud de elementos y, posteriormente, hacia la diferencia que ya podrá ser apreciada debido a la autorreflexión y al autoanálisis. Es decir, que el aula de Música puede considerarse un punto de encuentro cultural (De Moya, Hernández y Hernández, 2010) y de trabajo de los principales valores de la educación intercultural, como son el respeto a otras música y la tolerancia ante el resultado musical "del otro". Se pretende que el alumnado sea competente interculturalmente, aunque también culturalmente; de hecho, autores como Santos, Lorenzo y Priegue (2013) consideran que quizá la competencia cultural y la intercultural sean lo mismo.

En esta aula, gracias a su enfoque desde la perspectiva intercultural que pretende construir una sociedad donde la diferencia cultural sea considerada un factor de riqueza y no de división (Muñoz y Menchén, 2002), se logrará un reconocimiento por la diferencia y por la similitud que permitirán comprender al otro y construir una cultura común. Esa construcción será posible gracias al trabajo cooperativo (Leiva, 2011) y a las prácticas creativas características de la educación musical, que posibilitarán que esta clase se convierta en un espacio de trabajo de la interculturalidad.

Es muy importante que la Historia de la Música, la partitura o la improvisación musical, se conviertan en herramientas de búsqueda de similitudes musicales, más que un trabajo de búsqueda de nuevos elementos. No debe insistirse en señalar la diferencia (Touriñán, 2008) porque se puede desarrollar un reduccionismo, la pervivencia del poder de la cultura dominante que no es "la diferente"; sino más bien debe insistirse en buscar elementos comunes para luego analizar las posibles diferencias que ya no serán comprendidas de una forma tan lejana a las propias porque nunca debe olvidarse que la educación intercultural fomentará la cohesión social tan necesaria para comprender al otro (Borrero, 2012). Es obvio que el mundo es una marea de gente heterogénea, pero al mismo tiempo y de 
acuerdo con el cantante Macaco, todos "vibramos al mismo compás", y éste debería ayudar a crear una nueva cultura compartida.

La Música, a través del estudio de sus características estilísticas, con la interpretación, con el análisis, o bien con las improvisaciones, permite estudiar al otro desde un punto de vista ameno, y también permite conocerse mejor, hecho que ayudará a comprender cuán parecidos somos. La interculturalidad a través de la Música permite encontrar valores universales compartidos, dialogar sobre ellos y sobre la igualdad entre las personas (De Moya, Hernández y Hernández, 2010). Al fin y al cabo, la Música muestra fusiones de diferentes elementos procedentes de distintas zonas del mundo y nadie se extraña ante este hecho; transmite e invita a sentir emociones que no pueden considerarse exclusivas de una u otra cultura, y de nuevo nadie se extraña ante esos sentimientos universales compartidos... Entonces, ¿por qué extrañarse ante el hecho de que somos más iguales que diferentes? Desde esta perspectiva, la clase de Música debe organizar sus elementos característicos de un modo concreto para garantizar que el proceso educativo intercultural pueda tener lugar, verdaderamente.

\section{Metodología para el aula musical intercultural en Secundaria}

En primer lugar, ¿cómo hay que organizar la distribución espacial del aula? Ante todo, el aula musical deberá ser única, es decir, el alumnado no debería desplazarse a otros espacios para realizar interpretaciones instrumentales porque si se trasladan a otra aula ocupada sólo por el instrumental se está dejando ver un planteamiento organizativo "dividido": es decir, la teoría musical va por un lado y la práctica, por otro, cuando no deberían realizarse separadamente porque así no se contribuye a un proceso educativo globalizado y entendido como transmisor de valores interculturales. Esta organización en dos clases diferentes no contribuye a mostrar esta aula como un punto de encuentro, de experimentación, de intercambio, en el que la práctica de teoría-historia está estrechamente ligada a la práctica interpretativa y a los valores que ambas transmiten al estudiante. El mantenimiento de esa separación no contribuye al desarrollo global del educando, sino a mostrarle que esta clase musical es otro espacio más en el que la teoría del libro de texto rige las sesiones, mientras que la práctica es concebida como una "puntuación separada". Por supuesto, no todos los centros cuentan con aulas separadas, pero es una tónica bastante común.

No obstante, aunque el instrumental esté incluido en una única aula, éste no debe quedar distribuido al fondo, con dos espacios diferenciados (uno de explicación-mutismo del alumno, y otro de interpretación-conversación del alumnado) nuevamente; sino que el educando debería estar siempre en contacto con los instrumentos porque así se le transmiten dos ideas interculturales básicas: la disposición orquestal muestra que todos los instrumentos son decisivos y tienen mucho que aportar, estén donde estén localizados; y el hecho de que la interpretación y el análisis de los estilos a partir de las partituras que se van a interpretar es una forma más efectiva de comprender las diferentes características que también son las "nuestras". De forma que el espacio-tiempo para la explicación teórica quedaría "localizado" en el análisis interpretativo previo de la partitura que se señalaría y comentaría comparadamente al tiempo que se interpreta.

Si esta clase se orientase al aprendizaje de la Música desde una perspectiva intercultural, en la que el análisis de características musicales se enfocase como punto de partida para comprender las similitudes y las posibles diferencias entre culturas, no sólo estarían aprendiendo contenidos conceptuales sino además toda una serie de elementos transversales que les llevarían a comprender a las otras culturas, al mismo tiempo que a sí 
mismos. Es decir, debería enfocarse la clase de Música como un punto de encuentro, experimentación, improvisación, intercambio y reflexión comparada, alejado del libro de texto con sus tradicionales actividades sin espacio para la creatividad que alejan al alumnado de la base de la educación intercultural (el intercambio que promueven elementos como la composición y la improvisación musicales). Leiva (2011) apoyaría esta propuesta de un currículo alejado del uso exclusivo del libro de texto, al considerar que educar interculturalmente para evitar la exclusión implica ese distanciamiento, porque la interculturalidad necesita de espacios creativos que favorezcan la comunicación y, por tanto, el diálogo. En esta línea, la educación musical puede permitir estas ideas si recurre a herramientas como la improvisación y la composición (aunque sea muy básica).

En segundo lugar, ¿cómo deben organizarse los pupitres y el reparto instrumental? Mucho se ha escrito sobre la distribución de los pupitres en ESO (en círculo, en forma de "U"...), aunque para trabajar interculturalmente lo más adecuado sería un planteamiento que es propio de la actividad musical: la orquesta. Aunque se cuente sólo con carrillones, xilófonos y metalófonos, lo más adecuado es que se dispongan en un semicírculo y en primera posición estén los carrillones, después los xilófonos y luego los metalófonos; pero si se cuenta con instrumental de pequeña percusión (panderos, cascabeles, claves, güiros, cajas chinas) se colocarían al fondo y por sonoridades agudas a graves. Se podría considerar que así no todos "son igual", sin embargo en Música no se observa sea puntualización porque todos los instrumentos son imprescindibles para la interpretación sin que su posición espacial sea relevante; porque cada instrumento aporta una sonoridad especial sin la cual la partitura perdería aquello que la hace única y diferente; y porque la distribución espacial (instrumentos más graves al fondo y los más agudos delante) tiene un sentido y una razón puramente musicales que es determinante para el resultado final.

Entonces, el educando estará siempre ante su instrumento que, por supuesto, debe ir cambiando cada semana para que se trabaje el valor de compartir con "el otro", así como de respetar los materiales que todos deben disfrutar. El docente presentará las características de cada período o de cada artista mientras el alumnado cuenta con una partitura para proceder a la búsqueda de cada elemento comentado; se trataría de comentarlos y de buscar similitudes con canciones de diferentes procedencias y estilos.

En tercer lugar, ¿cómo debería organizarse el desarrollo del currículo? Los contenidos que el docente desarrolle en el aula deben "limitarse" a la presentación de características de los diferentes períodos histórico-musicales y al estudio de las principales figuras, tanto de la música clásica como de la música tradicional o popular. Por ejemplo, no sólo pueden trabajarse las características de la música romántica con Chopin, ya que se puede aprender a buscar elementos de ésta en la música tradicional polaca de la misma época e, incluso, en la música polaca moderna actual. Cada elemento estilístico que se trabaje debe tratar de localizarse en diferentes tipos de Música de diversa procedencia; además deberían analizarse las letras o buscarse las emociones o sentimientos que afloran en el educando durante la audición, o bien en los valores que le sugiera la combinación de armonías propuesta por el compositor. Siempre será conveniente para profundizar en las características de la interculturalidad, promover la búsqueda de otras música que puedan producir mensajes similares mediante las nuevas tecnologías de la información, así se promueve la adquisición de otras competencias como la de aprender a aprender y la digital.

\section{Conclusiones y discusión de los planteamientos}

Una organización intercultural del aula de Música, tanto en su espacio como en sus contenidos, permitirá adquirir una serie de valores como el respeto a la producción del otro, 
el respeto al silencio que implica escuchar al otro, la apreciación de los elementos propios como elementos enriquecedores. Por supuesto, es necesario trabajar otras músicas porque deben evitarse las proféticas palabras de Baba Dioum (citado en Venturi, 2004) que afirma que sólo se conserva lo que se quiere, sólo se quiere lo que se entiende, y sólo se entiende lo que te enseñan. De modo que es importante trabajar otras músicas para reflexionar culturalmente, para intercambiar elementos y fundirlos en un nuevo resultado, sin caer en el mero proceso de memorización que sólo resulta enriquecedor a nivel multicultural, pero no intercultural.

Se puede decir que hay cierta problemática en torno a la forma de educar a la pluriculturalidad debida a la falta de formación del futuro profesorado en este sentido. Desde nuestra experiencia como formadores de educadores, hemos podido comprobar cómo la falta de formación en esta temática ha llevado a que las páginas de recursos para profesorado estén llenas de actividades mal denominadas interculturales y multiculturales. Es precisamente este tipo de cuestiones las que llevan a seguir insistiendo en la necesidad de mostrar cómo es necesario recopilar formas de organizar los espacios de una forma intercultural para que el profesorado pueda atender adecuadamente a la diversidad del aula.

Debe conseguirse que la educación intercultural se convierta en el eje vertebrador de la clase musical. Entonces sí podrá decirse que el centro educativo es un espacio de trabajo y consecución de la interculturalidad, con todo lo que ésta implica en cuanto a relaciones, intercambio de experiencias, respeto y aprecio por el otro. Puede decirse que esta asignatura de Secundaria, tanto por los contenidos como por el desarrollo madurativo que se vivencia, es el espacio idóneo para que el alumnado tome conciencia sobre lo importante que es considerarse ciudadano del lugar que se habita y ciudadano del lugar del que se viene (FETE-UGT, 2011); de forma que los valores "prestados" y conocimientos de ambas ciudadanías le enriquezcan y le hagan mejor ciudadano "de aquí" y "de alli". Se trata de comprender la interculturalidad como un fenómeno positivo y de enriquecimiento de la convivencia (Rodríguez, Gallego, Sansó, Navarro, Velicias y Lago, 2011).

Se ha considerado que la interpretación de esta asignatura como un espacio de trabajo diferente al del resto de materias del centro puede resultar polémica y controvertida. Aunque algunos autores como De Moya, Hernández y Hernández (2010), han considerado que el aprendizaje de la Música debe centrarse en el componente social y dejar de lado la tendencia tradicionalista academicista (añadamos que en aras de un bien mayor). Se ha partido de una consideración muy lógica y que no menosprecia o disminuye la importancia que los contenidos teóricos pueden tener.

Si se organizan los contenidos musicales estableciendo relaciones con una serie de actitudes interculturales y sus respectivos procedimientos en el mismo sentido, no sólo se desarrollará en el alumnado un aprendizaje teórico musical sino también la adquisición de diferentes competencias que serán imprescindibles para su desarrollo social. No puede olvidarse que en la música destaca su función social y de conocimiento cultural (De Moya, Hernández y Hernández, 2010), ya que su práctica marcada por la cooperatividad, las interrelaciones y la interdependencia, la convierten en una herramienta social por excelencia dentro de las actividades que pueden realizarse y proponerse en cualquier centro educativo. $Y$ precisamente por esas características comentadas, el espacio educativo musical debe enfocarse a la adquisición de un objetivo principal como es la competencia intercultural. Esa interculturalidad que puede desarrollarse tan fácilmente aquí, es muy necesaria para la sociedad actual, caracterizada por el culto a la inmediatez y a la homogeneidad. Esta interculturalidad debe ser entendida como una reflexión profunda sobre las oportunidades que ofrece la diversidad cultural como elemento de intercambio de 
valores y actitudes (Leiva, 2008) que tan fácilmente puede desarrollarse gracias a todos los elementos que caracterizan la Música (como proceso educativo e interpretativo).

La educación musical es un proceso tan global que contribuye como ninguna otra a la formación de ciudadanos libres, democráticos y respetuosos con "el otro"; ya que es fundamental en el desarrollo integral del alumnado (De Moya, Hernández y Hernández, 2010) porque comprende otros ámbitos que pueden trabajarse en el centro educativo. Sin embargo, la última etapa obligatoria deberá convertirse en el punto final de construcción de una ciudadanía intercultural que se caracterice por asumir la existencia de colectivos distintos que construyan un proyecto común cohesionado (Folgueiras, Massot y Sabariego, 2008); y todo esto se verá facilitado por las características intrínsecas de esta asignatura en Secundaria. No obstante, nada de esto será posible si los educadores no están formados para mostrar cómo entre las diversas culturas que habitan el territorio existe una interconexión real; interconexión que se vuelve una realidad cuando se estudia la asignatura de Música.

En definitiva y aunque el aula de Música no se convierta en un espacio único y completo de trabajo de actitudes, valores y competencias, resulta lícito considerar que esta organización que se ha propuesto permitirá a los docentes trabajar la interculturalidad. Y al mismo tiempo, se ha planteado una clase de Música que permita la reconstrucción cultural compartida para mostrar que las culturas son abiertas y van cambiando (FETE-UGT, 2011), que la identidad es un proceso dinámico y en movimiento continuo (Samper, 2011); y esta realidad aparece perfectamente reflejada y recogida a lo largo de toda la Historia de la Música, de ahí la importancia de utilizarla como instrumento educativo intercultural y desde los planteamientos propuestos.

\section{Implicaciones socioeducativas y de investigación}

El docente cuando muestra un elemento estilístico concreto (concepto) que los alumnos deben buscar en la partitura con su interpretación (procedimiento) estará intentando mostrar qué implica o implicó dicha característica en el pensamiento de la época, qué supone para ellos hoy día de acuerdo con su contexto, si lo pueden encontrar en su música cotidiana o si es común a otras músicas (actitudes). Con este trabajo diario en el aula musical no puede más que concluirse cada unidad didáctica así trabajada con una recogida de los resultados observados en clase o con un test en el que se evalúe su grado de asimilación de los valores interculturales y de las competencias básicas (la competencia intercultural, principalmente). En Educación Infantil se evalúa al alumnado a través de la observación directa y de la recogida de información que implica, junto con los materiales que van realizando, y los maestros tienen perfectamente claro quién avanza y quién no. ¿Por qué en Secundaria no es posible esta opción? ¿Acaso ese tipo de evaluación no garantiza la comprobación de la adquisición de las diferentes competencias, objetivos y contenidos incluidos en la legislación vigente?

Realmente, este tipo de trabajo comentado puede contribuir a educar al alumnado desde y para la consecución de una situación de respeto entre culturas en el aula. El tipo de valores trabajados a través de la música les permitirá extrapolarlos fuera de la "asignatura musical" y vivenciarlos como una posibilidad en su devenir social.

Un interesante ejemplo de desarrollo didáctico intercultural que mostraría cómo se puede conseguir todo lo anteriormente comentado, desde el trabajo de las características del Romanticismo, se iniciaría con el comentario de una característica como es la de la exaltación del carácter nacional desde la interpretación de una pieza española, ya que 
permite amplias opciones de trabajo de valores y de otras culturas debido a los siglos de compartir territorio con otras culturas (musulmana y judía, principalmente). Entonces, se podrían establecer relaciones con otras músicas con la diferencia de que esas comparaciones deben ampliarse a los valores y emociones que se traslucen debido a la organización específica del material musical; es decir, se buscan puntos de contacto musicales que luego puedan ampliarse a otros niveles que les puedan influir en su desarrollo social. Ese trabajo que parte de la Música propia ayuda a conocer la cultura de pertenencia, comprendida como punto de partida para un análisis de elementos similares con la de otras culturas, y para garantizar un intercambio, una reflexión sobre los valores, sentimientos y emociones que pueden contener.

La inclusión de contenidos musicales de otras culturas no debe quedarse en una aproximación folclorista; igual que el estudio de la música tradicional de cada comunidad no debe convertirse en un instrumento político, sino que el estudio de ambas debe convertirse en un estudio comparado que permita aproximarse a las similitudes para comprender la igualdad entre ellas y, sólo después, las enriquecedoras diferencias.

Se debe promover una educación para aprender a vivir juntos (Touriñán, 2008), y añadamos, revueltos. El objetivo principal de todo proceso educativo debería ser garantizar el desarrollo humano, personal y social del educando dentro y fuera del centro educativo; de manera que pueda convivir, compartir e intercambiar experiencias, pensamientos y sentimientos, para construir un nuevo espacio común y compartido en igualdad de condiciones.

Si en el aula musical se cumpliesen estas ideas básicas se podrían evitar las actividades denominadas "interculturales" que se desarrollan de forma puntual debido a investigaciones o actuaciones concretas, que confunden la interculturalidad con el folklorismo. Se trataría de incorporar repertorios de culturas tradicionales desechadas (Samper, 2011), al tiempo que se suman a las obras clásicas y populares modernas, que quizá sean las que más unan al alumnado procedente de diferentes partes del mundo.

\section{Bibliografía}

Barguín, A., Alzola, N., Madinabeitia, M. y Urizar, A. (2010). El pensamiento del alumnado de magisterio sobre la inmigración y los inmigrantes. Revista Electrónica Interuniversitaria de Formación del Profesorado, 13 (4), 95-112.

Beriain, J. (2013). Encuentros con la alteridad e identidades múltiples. Árbor, 189 (761), 1-12.

Colectivo Yedra (2009). Convivencia escolar. Madrid: Cyan, Proyectos y Producciones Editoriales, S. A.

Borrero, R. (2012). Educación para todos y todas. Análisis del modelo educativo-social para una Educación Intercultural. Educatio Siglo XXI, 30 (2), 333-364.

Cruz Roja Española (2009). Cuaderno de actividades. Una propuesta para trabajar la Interculturalidad. Madrid: Cruz Roja Española.

De Moya, $M^{a}$ V., Hernández, J. R. y Hernández, J. A. (2010). Experiencia intercultural en el aula "La música nos une". Música y Educación, 84, 18-28.

Escudero, J. P. (2012). Interculturalidad e integración social en el aula a través del flamenco y los medios audiovisuales. Orientaciones y propuestas didácticas. DEDiCA. Revista de Educaçao e Humanidades, 3, 259-270. 
Etxeberría, F., Karrera, I. y Muruca, H. (2010). Competencias interculturales del profesorado con alumnado inmigrante en el País Vasco. Revista Electrónica Interuniversitaria de Formación del Profesorado, 13 (4), 79-94.

FETE-UGT (2009). La gestión de centros interculturales. Guía del Profesorado. Madrid: Cyan, Proyectos y Producciones Editoriales S. A.

FETE-UGT (2011). 20 Ideas para construir la interculturalidad y prevenir el racismo en educación. Madrid: Secretaría de Políticas Sociales de FETE-UGT y Departamento de Migraciones Confederal de UGT.

Folgueiras, P., Massot, I. y Sabariego, M. (2008). La ciudadanía activa e intercultural en alumnado de la ESO. Revista Interuniversitaria de Formación del Profesorado, 27 (11), $19-28$.

Fundación Secretariado Gitano (2007). Retos en los contextos multiculturales. Competencias interculturales y resolución de conflictos. Madrid: Fundación Secretariado Gitano.

Gómez, R. (2011). Música étnica, música intercultural. Un acercamiento en la Educación Primaria. Chile: Asociación por la Innovación Educativa Eduinnova.

González, O. (2010). Una experiencia de curriculum musical intercultural. Música y Educación, 81, 18-33.

Leiva, J. J. (2008). Interculturalidad, gestión de la convivencia y diversidad cultural en la escuela: un estudio de las actitudes del profesorado. Revista Iberoamericana de $\begin{array}{llll}\text { Educación, } & 46 & \text { (2). } & \text { Recuperado de }\end{array}$ http://www.rieoei.org/deloslectores/2297Leiva2.pdf

Leiva, J. J. (2011). La educación intercultural: un compromiso educativo para construir una escuela sin exclusiones. Revista Iberoamericana de Educación, 56 (1). Recuperado de http://www.rieoei.org/deloslectores/4242Olivencia.pdf

Lorenzo, O. y Espejo, J. A. (2011). Folclore musical en las aulas de primaria y secundaria. Música y Educación, 87, 36-47.

Pérez, G. y Sarrate, $M^{a}$ L. (2013). Diversidad cultural y ciudadanía. Hacia una educación superior inclusiva. Educación XX1, 16 (1), 85-104.

Pliego, N. y Valero, $M^{a}$ (2011). Alumnos inmigrantes en España: una realidad creciente. Hekademos. Revista Educativa Digital, 8, 77-90.

Rodríguez, H., Gallego, B., Sansó, C., Navarro, J. L., Velicias, M. y Lago, M. (2011). La educación intercultural en los centros escolares españoles. Revista Electrónica Interuniversitaria de Formación del Profesorado, 14 (1), 101-112.

Ruiz, C. y Merino, D. (2009). La experiencia escolar de los hijos de inmigrantes marroquíes en los Centros de Educación Secundaria Obligatoria. Revista Electrónica Interuniversitaria de Formación del Profesorado, 12 (3), 87-97.

Ruiz, V. J. (2007). Música y Diversidad Socio-Cultural en ESO: La Composición Musical, un Punto de Encuentro. Recuperado de http://www.intercentres.edu.gva.es/iesleonardodavinci/premios-yreconocimientos/Musica-2007.pdf

Samper, A. (2011). Educación musical a nivel superior e interculturalidad en el siglo XXI: nuevas epistemologías, nuevas aproximaciones didácticas. El Artista, 8, 297-316. 
Sales, A. (2012). La formación intercultural del profesorado: estrategias para un proceso de investigación-acción. Educatio Siglo XXI, 30 (1), 113-132.

Santos, M. A., Lorenzo, M. y Priegue, D. (2013). (Red) conectando a los profesores para el desarrollo de la interculturalidad. Educación XX1, 16 (1), 63-84.

Touriñán, J. M. (2008). Educación en valores, educación intercultural y formación para la convivencia pacífica. La Coruña: Netbiblo.

UNESCO (2006). Guidelines on Intercultural Education. París: UNESCO.

Venturi, L. (2004). Historia de la crítica de arte. Barcelona: Random House Mondadori, S. A.

\section{Autora}

María del Mar Bernabé Villodre

Doctora en Teoría e Historia de la Educación (UMU), Máster en Musicoterapia (UCV), Título Superior de Música en Pedagogía del Lenguaje y la Educación Musical (CSMU), Licenciada en Historia del Arte (UMU). Colaboradora del Grupo de Investigación "Educación, calidad de vida y desarrollo" de la Universidad de Murcia. Profesora Ayudante del Dpto. Didáctica de la Expresión Musical, Plástica y Corporal (UV). Líneas de investigación centradas en didáctica musical, didáctica de las CC.SS. y pedagogía musical intercultural. 